\title{
Pulse Soliton Solutions of the Modified KdV and Born-Infeld Equations
}

\author{
Jean Roger Bogning \\ Department of Physics, Higher Teacher's Training College Bambili, University of Bamenda, Bamenda, Cameroon \\ Email: rbogning@yahoo.com,jrbogning@yahoo.fr
}

Received January 19, 2013; revised February 19, 2013; accepted March 26, 2013

Copyright (C) 2013 Jean Roger Bogning. This is an open access article distributed under the Creative Commons Attribution License, which permits unrestricted use, distribution, and reproduction in any medium, provided the original work is properly cited.

\begin{abstract}
In this work, we use the Bogning-Djeumen Tchaho-Kofané method to look for all solutions of shape Sech ${ }^{n}$ - of the modified KdV and Born-Infeld Equations. $n$ being a real number, we obtain the soliton solutions when $n$ is positive and the non soliton solutions when $n$ is negative.
\end{abstract}

Keywords: KdV Equation; Born-Infeld Equation; Soliton Solution; BDKm; Modified KdV Equation

\section{Introduction}

Dynamics of physical phenomena are more often described analytically by nonlinear partial differential equations (NPDEs). These equations are varied and are met in various branches of the Physics, notably in Classical Mechanics, Quantum Mechanics, Fluid Mechanics, Electrodynamics, and so on. These NPDEs are not always easy to solve. Knowing that all analytic solutions of a NPDE always bring supplementary information, many researchers develop every day the means to solve these NPDEs or to improve some existing solutions. It is in this optics that several methods and techniques facilitating the resolution of NPDEs have been proposed [1-3]. From all these equations, those which contain the scattering terms and nonlinear terms create a particular interest, because they generally admit some soliton solutions; the soliton being a futuristic concept which imposes itself progressively in the world of Physics and especially as alternative in the modern telecommunications. This propensity is translated besides by all recent works that have been published [4-7].

Among these numerous equations, two have drawn our attention in this work. It is precisely the equation of Born-Infeld developed by Born and Infeld in the years 1930 and admits numerous applications in physics [8-15]. The second is the equation of $\mathrm{KdV}$. This equation which is the first that describes the dynamics of the navy waves has been also the object of several investigations and modifications [16-22].

Abdul-Majid Wazwaz in one of his recent works dem- onstrated that the modified $\mathrm{KdV}$ equation admitted several types of solutions notably the solitons, the peakons and the cuspons [23]. In this work, we follow the same logic to look for all solutions of the shape $\operatorname{Sech}^{n}$ - of the modified $\mathrm{KdV}$ equation. We are also going to determine all solutions $\mathrm{Sech}^{n}$ - of the equation of Born-Infeld. The problem we want to solve has been motivated in the following manner: We know that the equation of $\mathrm{KdV}$ under its initial shape admits a solution in $\mathrm{Sech}^{n}$ - (for $n=2$ ) but we don't know what happens precisely when $n \neq 2$. Thus, we at first look for the equation of $\mathrm{KdV}$ under its initial shape then admit a solution of shape Sech $^{n}$ - with $n \neq 2$. But it is not the first objective of this work, we want to test the efficiency of the method rightly by using on a simplest case. The goal of work is to look for all pulse solutions of the shape $\mathrm{Sech}^{n}$ - in the modified $\mathrm{KdV}$ and Born-Infeld equations by the Bogning-Djeumen Tchaho-Kofané method (BDKm) [24-28].

This work is organized as follows: in Section 2, we verify if the solution in $\mathrm{Sech}^{n}$ - of the initial KdV equation is only obtained for $n=2$. Section 3 is devoted in research of all solutions in $\mathrm{Sech}^{n}$ - of the modified KdV equation. The same analysis is made in Section 4 for equation of Born-Infeld. Finally, in Section 5, we conclude our work.

\section{Sech ${ }^{n}$ - Soliton Solution of the Initial KdV Equation}

The $\mathrm{KdV}$ equation in its initial form is given by

$$
\partial_{t} \phi+\partial_{x}^{3} \phi+\eta \phi \partial_{x} \phi=0
$$


where $\phi$ represents the amplitude of navy wave, $t$ is the time and $x$ the spatial variable. The problem here is to construct the solution of Equation (1) in the form

$$
\phi(x, t)=\operatorname{asech}^{n} \alpha\left(x-V_{g} t\right),
$$

where $a$ is a constant and $n$ a real number which is different of two. When we introduce the Equation (2) in the Equation (1) we obtain an equation of which the use of the different transformations linked to BDKm [24-28] permits to write it under the form

$$
\begin{aligned}
& \sum_{i, j, n} F\left(a_{i j}\right) / \cosh ^{n} \alpha x+\sum_{i, j, m} G\left(a_{i j}\right) \sinh \alpha x / \cosh ^{m} \alpha x \\
& +\sum_{i, j, k} H\left(a_{i j}\right) \cosh ^{k} \alpha x+\sum_{i, j, l} T\left(a_{i j}\right) \cosh ^{l} \alpha x \sinh \alpha x \\
& +\sum_{i, j} W\left(a_{i j}\right)=0 .
\end{aligned}
$$

called equation of ranges where $F\left(a_{i j}\right), G\left(a_{i j}\right)$, $H\left(a_{i j}\right), T\left(a_{i j}\right)$ and $W\left(a_{i j}\right)$ are functions of the coefficients $a_{i j}$ to determine and $i, j, n, m, k, l$ are positive whole integers.

These transformations concern the terms obtain when we introduce Equation (2) in Equation (1) and they are given by the following equations

$$
\begin{aligned}
& (\tanh \alpha x)^{2 k}=\sum_{i=0}^{k}(-1)^{i} C_{k}^{i}(\operatorname{sech} \alpha x)^{2 i} \\
& (\tanh \alpha x)^{2 k+1}=\sum_{i=0}^{k}(-1)^{i} C_{k}^{i}(\operatorname{sech} \alpha x)^{2 i+1} \sinh \alpha x \\
& (\operatorname{sech} \alpha x)^{2 k+2}(\sinh \alpha x)^{2 k+1} \\
& =\sum_{i=0}^{k}(-1)^{i} C_{k}^{i}(\operatorname{sech} \alpha x)^{2 i+2} \sinh \alpha x \\
& (\operatorname{sech} \alpha x)^{2 k+2 n}(\sinh \alpha x)^{2 k+1} \\
& =\sum_{i=0}^{k}(-1)^{i} C_{k}^{i}(\operatorname{sech} \alpha x)^{2 i+2 n} \sinh \alpha x \\
& (\operatorname{sech} \alpha x)^{2 k-1}(\sinh \alpha x)^{2 k}=\sum_{i=0}^{k}(-1)^{i} C_{k}^{i}(\operatorname{sech} \alpha x)^{2 i-1}, \\
& (\operatorname{sech} \alpha x)^{2 k+1}(\sinh \alpha x)^{4 k+2} \\
& =\sum_{i=0}^{2 k+1}(-1)^{i} C_{2 k+1}^{i}(\operatorname{sech} \alpha x)^{2 i-2 k-1} \\
& (\operatorname{sech} \alpha x)^{2 k}(\sinh \alpha x)^{2 k+1} \\
& =\sum_{i=0}^{k}(-1)^{i} C_{k}^{i}(\operatorname{sech} \alpha x)^{2 i} \sinh \alpha x \\
& (\operatorname{sech} \alpha x)^{4 k+2}(\sinh \alpha x)^{2 k+1} \\
& =\sum_{i=0}^{k}(-1)^{i} C_{k}^{i}(\operatorname{sech} \alpha x)^{2(i+k+1)}
\end{aligned}
$$

$$
\begin{aligned}
& (\operatorname{sech} \alpha x)^{2 k-1}(\sinh \alpha x)^{2 k+1}=\sum_{i=0}^{k}(-1)^{i} C_{k}^{i}(\operatorname{sech} \alpha x)^{2 i-1}, \\
& (\operatorname{sech} \alpha x)^{2 k+1}(\sinh \alpha x)^{2 k}=\sum_{i=0}^{k}(-1)^{i} C_{k}^{i}(\operatorname{sech} \alpha x)^{2 i+1}
\end{aligned}
$$

where $i, k \in \square, \alpha \in \square$ and $n$ are fixed integers such that $C_{k}^{i}=\frac{k !}{i !(k-i) !}$. Thus, substituting Equation (2) in Equation (1), we obtain with the help of the preceding transformations the general equation

$$
\begin{aligned}
& \left(n a \alpha V_{g}-\alpha^{3} n^{3} a\right) \frac{\sinh \alpha\left(x-V_{g} t\right)}{\cosh ^{n+1} \alpha\left(x-V_{g} t\right)} \\
& -\eta n a^{2} \alpha \frac{\sinh \alpha\left(x-V_{g} t\right)}{\cosh ^{2 n+1} \alpha\left(x-V_{g} t\right)} \\
& +n(n+1)(n+2) a \alpha^{3} \frac{\sinh \alpha\left(x-V_{g} t\right)}{\cosh ^{n+3} \alpha\left(x-V_{g} t\right)}=0 .
\end{aligned}
$$

After haven gotten the Equation (13), the principle of analysis is as follows: before passing to the identification of coefficients of the terms in

$$
\sinh \alpha\left(x-V_{g} t\right) / \cosh ^{i} \alpha\left(x-V_{g} t\right) \quad(i=n+1,2 n+1, n+3)
$$

of Equation (14), we verify if there exist some values of $n$ for which some terms of the Equation (14) have the same factor $\sinh \alpha\left(x-V_{g} t\right) / \cosh ^{i} \alpha\left(x-V_{g} t\right)$ ? So for the Equation (14), the factors

$$
\sinh \alpha\left(x-V_{g} t\right) / \cosh ^{2 n+1} \alpha\left(x-V_{g} t\right)
$$

and

$$
\sinh \alpha\left(x-V_{g} t\right) / \cosh ^{n+3} \alpha\left(x-V_{g} t\right)
$$

are identical for $n=2$ (it is sufficient to solve the equation $2 n+1=n+3)$. We also notice that the factors

$$
\sinh \alpha\left(x-V_{g} t\right) / \cosh ^{n+1} \alpha\left(x-V_{g} t\right)
$$

and

$$
\sinh \alpha\left(x-V_{g} t\right) / \cosh ^{2 n+1} \alpha\left(x-V_{g} t\right)
$$

are identical for $n=0$. But for $n=0$, the Equation (14) leads to a trivial solution of the type $\phi(x, t)=a$ (constant) that is not interesting. For $n=2$, Equation (14) is written as

$$
\begin{aligned}
& \left(2 a \alpha V_{g}-8 \alpha^{3} a\right) \frac{\sinh \alpha\left(x-V_{g} t\right)}{\cosh ^{3} \alpha\left(x-V_{g} t\right)} \\
& +\left(24 a \alpha^{3}-2 \eta a^{2} \alpha\right) \frac{\sinh \alpha\left(x-V_{g} t\right)}{\cosh ^{5} \alpha\left(x-V_{g} t\right)}=0 .
\end{aligned}
$$

From Equation (15), we obtain the following ranges of equations 


$$
12 \alpha^{2}-\eta a=0
$$

and

$$
V_{g}-4 \alpha^{2}=0
$$

The resolution of Equation (16) and Equation (17) permits to have $a=12 \alpha^{2} / \eta$ and $\alpha= \pm \sqrt{V_{g}} / 2$. While reporting these values obtained in the expression (2), we at first find a solution considered like particular solution of the Equation (1) and given by

$$
\phi(x, t)=\frac{3 V_{g}}{\eta} \operatorname{sech}^{2} \pm \frac{\sqrt{V_{g}}}{2}\left(x-V_{g} t\right)
$$

with $V_{g} \geq 0$ and $\eta \neq 0$. The particular cases $n=0$ and $n=2$ being studied, we now look at the case where $n \neq 0$ and $n \neq 2$. So for $n \neq 0$ and $n \neq 2$, the Equation (14) doesn't present more terms that can merge themselves. At this level, the only acceptable solution is the trivial solution $U(x, t)=0$ which doesn't have importance. In conclusion, the equation of $\mathrm{KdV}$ as considered above admits non trivial solutions only for $n=2$ and Equation (18) also gives the general solution of the $\mathrm{KdV}$ equation considered under its initial shape given by Equation (1).

\section{Sech ${ }^{n}$ - Soliton Solution of the Modified KdV Equation}

The modified $\mathrm{KdV}$ equation that we are going to use here is in the form [23]

$$
\begin{aligned}
& U U_{x x t}-U_{x} U_{x t}-4 U^{3} U_{t}+4 U U_{x x x} \\
& -4 U_{x} U_{x x}-16 U^{3} U_{x}=0 .
\end{aligned}
$$

We look for the solutions of Equation (19) under the shape

$$
U_{n}(x, t)=\operatorname{asech}^{n}\left(\alpha x-\alpha_{0} t\right),
$$

where $a$ and $\alpha$ are constants to be determined, $\alpha_{0}$ a known constant and $n$ a real number to determine. Thus, taking into account Equation (20) into Equation (19) gives with the help of transformations (4), (5), , , (13) the following ranges equation

$$
\begin{aligned}
& {\left[-a \alpha^{2} \alpha_{0}(n+1)(n+2)+a^{2} \alpha^{2} \alpha_{0} n^{2}(n+1)\right.} \\
& \left.+4 a^{2} \alpha^{3} n(n+1)(n+2)-4 a^{2} \alpha^{3} n^{2}(n+1)\right] \\
& \cdot \frac{\sinh \left(\alpha x-\alpha_{0} t\right)}{\cosh ^{2 n+3}\left(\alpha x-\alpha_{0} t\right)} \\
& +\left(16 a^{4} \alpha n-4 a^{4} n \alpha_{0}\right) \frac{\sinh \left(\alpha x-\alpha_{0} t\right)}{\cosh ^{4 n+1}\left(\alpha x-\alpha_{0} t\right)}=0 .
\end{aligned}
$$

The two terms of the Equation (21) merge themselves for $n=1$ (it is sufficient to solve the equation $2 n+3=4 n+1)$. So from the Equation (21), we obtain

$$
\left(8 \alpha-2 \alpha_{0}\right) a^{3}+\left(\alpha^{2} \alpha_{0}+8 \alpha^{3}\right) a-3 \alpha^{2} \alpha_{0}=0,
$$

with $a \neq 0$. Equation (22) can also be written as

$$
a^{3}+\left(\frac{\alpha^{2} \alpha_{0}+8 \alpha^{3}}{8 \alpha-2 \alpha_{0}}\right) a-\frac{3 \alpha^{2} \alpha_{0}}{8 \alpha-2 \alpha_{0}}=0
$$

with $\frac{\alpha}{\alpha_{0}} \neq \frac{1}{4}$. By setting $P=\frac{\alpha^{2} \alpha_{0}+8 \alpha^{3}}{8 \alpha-2 \alpha_{0}} \quad$ and $q=\frac{-3 \alpha^{2} \alpha_{0}}{8 \alpha-2 \alpha_{0}}$, Equation (23) becomes

$$
a^{3}+P a+q=0 .
$$

By using the Cardano's method, we set $a=u+v$ leads to the relations

$$
u^{3}+v^{3}=-q
$$

and

$$
u^{3} v^{3}=\frac{-P^{3}}{27}
$$

The resolution of the coupled system (25) and (26) gives

$$
a=\sqrt[3]{\frac{-q+\sqrt{\frac{27 q^{2}+4 P^{3}}{27}}}{2}}+\sqrt[3]{\frac{-q-\sqrt{\frac{27 q^{2}+4 P^{3}}{27}}}{2}} .
$$

Therefore the solution obtained for $n=1$ is given by

$$
\begin{aligned}
U_{1}(x, t) & =\left[\sqrt[3]{\frac{-q+\sqrt{\frac{27 q^{2}+4 P^{3}}{27}}}{2}}\right. \\
& \left.+\sqrt[3]{\frac{-q-\sqrt{\frac{27 q^{2}+4 P^{3}}{27}}}{2}}\right] \operatorname{sech}\left(\alpha x-\alpha_{0} t\right) .
\end{aligned}
$$

For $n \neq 1$, Equation (21) leads to the following relations:

$$
\begin{array}{r}
\text { Term in } \frac{\sinh \left(\alpha x-\alpha_{0} t\right)}{\cosh ^{4 n+1}\left(\alpha x-\alpha_{0} t\right)}, \\
\frac{\alpha}{\alpha_{0}}=\frac{1}{4} .
\end{array}
$$

Term in $\frac{\sinh \left(\alpha x-\alpha_{0} t\right)}{\cosh ^{2 n+3}\left(\alpha x-\alpha_{0} t\right)}$

$$
a=\frac{1}{n} \quad \text { with } n \neq 0, n \neq 1 .
$$

Then for $n \neq 0$ and $n \neq 1$, the solution of the modi- 
fied $\mathrm{KdV}$ equation is given by

$$
U_{n}(x, t)=\frac{1}{n} \operatorname{sech}^{n} \frac{\alpha_{0}}{4}(x-4 t) .
$$

In the following section, we are going to make an analogous survey for the equation of Born-Infeld.

\section{Sech ${ }^{n}$ - Solution of the Born-Infeld's Equation}

The Born-Infeld's equation that we want to analyze in this section is given by

$$
\left(1-U_{t}^{2}\right) U_{x x}+2 U_{x} U_{t} U_{x t}-\left(1+U_{x}^{2}\right) U_{t t}=0 .
$$

Like in the preceeding section, we look for the solution of Equation (31) in the form of Equation (20). With the help of the transformations (4), (5), $\cdots,(13)$, we obtain

$$
\begin{aligned}
& \frac{a\left(\alpha^{2}-\alpha_{0}^{2}\right) n^{2}}{\cosh ^{n}\left(\alpha x-\alpha_{0} t\right)}+\frac{a\left(\alpha_{0}^{2}-\alpha^{2}\right) n(n+1)}{\cosh ^{n+2}\left(\alpha x-\alpha_{0} t\right)} \\
& +\frac{2 \alpha^{2} \alpha_{0}^{2}\left(a^{2}-a^{3}\right) n^{4}}{\cosh ^{3 n}\left(\alpha x-\alpha_{0} t\right)} \\
& +\frac{4 \alpha^{2} \alpha_{0}^{2}\left(a^{3}-a^{2}\right) n^{2}+2 \alpha^{2} \alpha_{0}^{2}\left(a^{3}-a^{2}\right) n^{3}}{\cosh ^{3 n+2}\left(\alpha x-\alpha_{0} t\right)} \\
& +\frac{2 \alpha^{2} \alpha_{0}^{2}\left(a^{2}-a^{3}\right) n^{3}(n+1)}{\cosh ^{3 n+4}\left(\alpha x-\alpha_{0} t\right)}=0 .
\end{aligned}
$$

As in the previous cases, we first look for the values of $n$ for which the terms which constituted Equation (33) merge themselves. Thus, the terms in

$1 / \cosh ^{n+2}\left(\alpha x-\alpha_{0} t\right)$ and $1 / \cosh ^{3 n+2}\left(\alpha x-\alpha_{0} t\right)$ merge for $n=0$ as well as the terms in $1 / \cosh ^{n}\left(\alpha x-\alpha_{0} t\right)$ and $1 / \cosh ^{3 n}\left(\alpha x-\alpha_{0} t\right)$. The terms in $1 / \cosh ^{n+2}\left(\alpha x-\alpha_{0} t\right)$ and $1 / \cosh ^{3 n+4}\left(\alpha x-\alpha_{0} t\right)$ as well as the terms in $1 / \cosh ^{3 n+2}\left(\alpha x-\alpha_{0} t\right)$ and $1 / \cosh ^{n}\left(\alpha x-\alpha_{0} t\right)$ merge for $n=-1$. The terms in $1 / \cosh ^{n}\left(\alpha x-\alpha_{0} t\right)$ and $1 / \cosh ^{3 n+4}\left(\alpha x-\alpha_{0} t\right)$ merge for $n=-2$. The terms in $1 / \cosh ^{n+2}\left(\alpha x-\alpha_{0} t\right)$ and $1 / \cosh ^{3 n}\left(\alpha x-\alpha_{0} t\right)$ merge

for $n=1$. Then, for $n \in\{-2,-1,0,1\}$, the Equation (33) contains the terms which merge. In the continuation we are going to study in detail the Equation (33) when $n$ is equal to these found values.

- For $n=-2$, the resolution of Equation (33) gives $\alpha= \pm \alpha_{0}$ and $a=1$. The solution of Equation (32) in this case is given by

$$
\begin{aligned}
U_{-2}(x, t) & =\operatorname{sech}^{-2}\left[ \pm \alpha_{0}(x-t)\right] \\
& =\cosh ^{2}\left[ \pm \alpha_{0}(x-t)\right] .
\end{aligned}
$$

- For $n=-1$, the resolution of Equation (33) gives $\alpha= \pm \alpha_{0}$ and $a=1$. The solution of Equation (32) in this case is given by

$$
\begin{aligned}
U_{-1}(x, t) & =\operatorname{sech}^{-1}\left[ \pm \alpha_{0}(x-t)\right] \\
& =\cosh \left[ \pm \alpha_{0}(x-t)\right] .
\end{aligned}
$$

- For $n=0$, we obtain $U_{0}(x, t)=a$ which is a constant.

- For $n=1$, the Equation (33) becomes

$$
\begin{aligned}
& \frac{4 a\left(\alpha^{2}-\alpha_{0}^{2}\right)}{\cosh \left(\alpha x-\alpha_{0} t\right)}+\frac{2 a\left(\alpha^{2}-\alpha_{0}^{2}\right)+2 \alpha^{2} \alpha_{0}^{2}\left(a^{2}-a^{3}\right)}{\cosh ^{3}\left(\alpha x-\alpha_{0} t\right)} \\
& +\frac{6 \alpha^{2} \alpha_{0}^{2}\left(a^{3}-a^{2}\right)}{\cosh ^{5}\left(\alpha x-\alpha_{0} t\right)}+\frac{4 \alpha^{2} \alpha_{0}^{2}\left(a^{2}-a^{3}\right)}{\cosh ^{7}\left(\alpha x-\alpha_{0} t\right)}=0 .
\end{aligned}
$$

While equating the terms of the Equation (36) to be zero we obtain $\alpha= \pm \alpha_{0} ; a=1$ and the solution of the Equation (32) is

$$
U_{1}(x, t)=\operatorname{sech}\left[ \pm \alpha_{0}(x-t)\right] .
$$

In the case where $n \neq\{-2,-1,0,1\}$, we identify the terms of Equation (32) to zero and look for the non trivial solutions $(a \neq 0)$ which give $\alpha= \pm \alpha_{0}$ and $a=1$. So we can affirm without hesitation that the general solution of the equation of Born-Infeld is

$$
\begin{aligned}
& U_{n}(x, t)=\operatorname{sech}^{n}\left[ \pm \alpha_{0}(x-t)\right], \\
& \forall n \in \square .
\end{aligned}
$$

\section{Conclusion}

In this work, we constructed with success the solutions of shape $\mathrm{Sech}^{n}$ - of the KdV and Born-Infeld equations. Our survey has been guided by the fact that, we already know that by a direct integration, the initial $\mathrm{KdV}$ equation admits a solution in $\operatorname{Sech}^{n}-(n=2)$ and we don't know what happens precisely when $n \neq 2$. To this effect, we supposed that its solution is under the shape $\operatorname{Sech}^{\mathrm{n}}$ - to arrive at the conclusion $n=2$. The survey has been extended to the case of the modified KdV and Born-Infeld equations. The solutions of shape

$$
U_{n}(x, t)=\frac{1}{n} \operatorname{sech}^{n} \frac{\alpha_{0}}{4}(x-4 t),
$$

for $n \neq 0, n \neq 1$ and $U_{n}(x, t)=\operatorname{sech}^{n} \pm \alpha_{0}(x-t)$,

$\forall n \in \square$, have been obtained respectively. The success of this survey is due to the BDKm whose mastery permits to push the analysis as far as possible in the equations that present an elevated nonlinearity as seen in the two equations studied scrupulously. 


\section{Acknowledgements}

I acknowledge support from the ministry of Higher Education of Cameroon through its program of support to Research, which enabled me to carry out this work. I also thank Mr Tsapgou Jean Jeremie for some corrections after the reading of the work.

\section{REFERENCES}

[1] R. Hirota, "The Direct Method in Soliton Theory," Cambridge University Press, Cambridge, 2004. doi:10.1017/CBO9780511543043

[2] W. Herman and A. Nuseir, "Symbolic Methods to Construct Exact Solutions of Nonlinear Partial Differential Equations," Mathematics and Computers in Simulation, Vol. 43, No. 1, 1997, pp. 13-27. doi:10.1016/S0378-4754(96)00053-5

[3] A. M. Wazwaz, "Soliton Solutions for Two $(3+1)$-Dimensional Non Integrable KdV-Type Equations," Mathematical and Computer Modelling, Vol. 55, No. 5-6, 2012, pp. 1845-1848. doi:10.1016/j.mcm.2011.11.082

[4] A. M. Wazwaz, "Two Forms of $(3+1)$-Dimensional BType Kadomtsev-Petviashvili Equation: Multiple Soliton Solutions," Physica Scripta, Vol. 86, No. 3, 2012, pp. 035007-035015. doi:10.1088/0031-8949/86/03/035007

[5] Q. C. Jiang, Y. L. Su and X. M. Ji, "Coupling Effects of Grey-Grey Separate Spatial Screening Soliton Pairs," Physica Scripta, Vol. 86, No. 3, 2012, pp. 035404-035408. doi:10.1088/0031-8949/86/03/035404

[6] T. Kaladze, S. Mahmood and H. Ur-Rehman, "Acoustic Nonlinear Periodic (Cnoidal) Waves and Solitons in Pair-Ion Plasmas," Physica Scripta, Vol. 86, No. 3, 2012, pp. 035506-035514. doi:10.1088/0031-8949/86/03/035506

[7] Q.-Y. Chen, P. G. Kevrekidis and B. A. Malomed, "Dynamics of Bright Solitons and Soliton Arrays in the Nonlinear Schrödinger Equation with a Combination of Random and Harmonic Potentials," Physica Scripta, Vol. 2012, No. T149, 2012, pp. 014001-014007. doi:10.1088/0031-8949/2012/T149/014001

[8] M. Born and L. Infeld, "1934 Foundations of the New Field Theory," Proceedings of the Royal Society A, Vol. 144, No. 852, 1934, pp. 425-451.

[9] M. Born, "On the Quantum Theory of Electromagnetic Field," Proceedings of the Royal Society A, Vol. 143, No. 849, 1934, pp. 410-437.

[10] D. I. Blokhintsev, "Space and Time in the Microcosm," Nanka, Moscow, 1982.

[11] M. KöiV and V. Rosenhaus, "Family of Two Dimensional Born-Infeld Equations and a System of Conservation Laws," IZV. Akad. Nauk Est. SSR. Fizika, MathematiKa, Vol. 28, No. 3, 1979, pp. 187-193.

[12] B. M. Barbashov and N. A. Chernikov, "Solving and Quantization of Nonlinear Two-Dimensional Model Born-Infeld Type," Zhurn EKsperin I Teor Fiziki, Vol. 60 No. 5, 1966, pp. 1926-1308.

[13] B. M. Barbashov and N. A. Chernikov, "Interaction of
Two Plane Waves in Born-Infeld Electrodynamics," Fizika Vysokikh Energii I Teoria Elementarnykh Chastitz, Kyiv, 1967, pp. 733-743.

[14] W. I. Fushchych and V. A. Tychinin, "On Linearization of Some Nonlinear Equations with the Help of Non Local Transformations," Institute of Mathematics, Academic of Sciences, Ukraine, 1982.

[15] V. Fedorchuk, "Symmetry Reduction and Exact Solutions of the Euler-Lagrange-Born-Infeld, Multidimensional Monge-Ampere and Eikonal Equations," Journal of Nonlinear Mathematical Physics, Vol. 2, No. 3-4, 1995, pp. 329-333.

[16] R. K. Bullough, “The Wave Par Excellence: The Solitary Progressive Great Wave of Equilibrium of the Fluid and Early History of the Solitary Wave," Springer, New York, 1988, pp. 150-281.

[17] J. de Frutos and J. M. Sanz-Serna, "Accuracy and Conservation Properties in Numerical Integration. The Case of the Korteweg-de Vries Equation," Numerische Mathematik, Vol. 75, No. 4, 1997, pp. 421-445. doi: $10.1007 / \mathrm{s} 002110050247$

[18] J. S. Russel, "Report on Waves: Report of the $14^{\text {th }}$ Meeting of the British Association for the Advancement of Science," York, 1884, pp. 314-390.

[19] W. E. Schiesser, "Method of Lines Solution of the Korteweg-de Vries Equation," Computers \& Mathematics with Applications, Vol. 28, No. 10-21, 1994, pp. 147-154.

[20] E. Varley and B. R. Seymour "A Simple Derivation of the N-Soliton Solutions to the Korteweg-de Vries Equation," SIAM: SIAM Journal on Applied Mathematics, Vol. 58, No. 3, 1998, pp. 904-911. doi: $10.1137 / \mathrm{S} 0036139996303270$

[21] D. Vvedenskii, "Partial Differential Equations with Mathematica," Wokingham Addison, Wesley, 1992.

[22] S. Wolfram, "The Mathematica Book. Cambridge (UK)," Cambridge University Press, 1999.

[23] A. M. Wazwaz, "A Modified KdV-Type Equation That Admits a Variety of Travelling Wave Solutions: Kinks, Solitons, Peakons and Cuspons," Physica Scripta, Vol. 86, No. 4, 2012, pp. 045501-045506. doi:10.1088/0031-8949/86/04/045501

[24] C. T. Djeumen Tchaho, J. R. Bogning and T. C. Kofane, "TC 2010 Construction of the Analytical Solitary Wave Solutions of Modified Kuramoto-Sivashinsky Equation by the Method of Identification of Coefficients of the Hyperbolic Functions," Far East Journal of Dynamical Systems, Vol. 14, No. 1, 2010, pp. 14-17.

[25] C. T. Djeumen Tchaho, J. R. Bogning and T. C. Kofane, "Multi-Soliton Solutions of the Modified Kuramoto-Sivashinsky's Equation by the BDK Method," Far East Journal of Dynamical Systems, Vol. 15, No. 2, 2011, pp. 83-98.

[26] J. R. Bogning, C. T. Djeumen Tchaho and T. C. Kofané, "Construction of the Soliton Solutions of the GinzburgLandau Equations by the New Bogning-Djeumen Tchaho-Kofané method," Physica Scripta, Vol. 85, No. 2, 2012, pp. 025013-025017. doi:10.1088/0031-8949/85/02/025013

[27] C. T. Djeumen Tchaho, J. R. Bogning, and T. C. Kofané, 
"Modulated Soliton Solution of the Modified KuramotoSivashinsky's Equation," American Journal of Computational and Applied Mathematics, Vol. 2, No. 5, 2012, pp. 218-224. doi:10.5923/j.ajcam.20120205.03

[28] J. R. Bogning, C. T. Djeumen Tchaho and T. C. Kofané,
"TC 2012 Generalization of the Bogning-Djeumen Tchaho-Kofane Method for the Construction of the Solitary Waves and the Survey of the Instabilities," Far East Journal of Dynamical Systems, Vol. 20, No. 2, 2012, pp. 101-119. 